\title{
Design of Coprime DFT Arrays and Filter Banks
}

\author{
Chun-Lin Liu and P. P. Vaidyanathan \\ Dept. of Electrical Engineering, 136-93 \\ California Institute of Technology, Pasadena, CA 91125, USA \\ cl.liu@caltech.edu,ppvnath@ systems.caltech.edu
}

\begin{abstract}
Coprime arrays offer degrees of freedom of $O(M N)$ given $O(M+N)$ sensors, where $M$ and $N$ are coprime integers. The performance of coprime arrays is based on coprime DFT filter banks (coprime DFTFBs), which cascade an $M$-channel DFTFB and an $N$-channel DFTFB to achieve $M N$-channel filter banks. However, practical designs of coprime DFTFBs have not been fully studied. In this paper, a systematic design is related to IFIR filter designs, based on $M, N$, filter orders, and peak ripples. Our design owns a parameter $\lambda$ that provides tradeoffs between passbands and stopbands. A design example for different $\lambda$ is also presented.
\end{abstract}

\section{INTRODUCTION}

Coprime DFT filter banks (coprime DFTFBs), introduced in [12], have applications in sensor array processing. Given two integers $M$ and $N$ that are coprime, two uniform linear arrays with interelement spacing $N \lambda / 2$ and $M \lambda / 2$, respectively, are designed properly to achieve $O(M N)$ degrees of freedom from $O(M+N)$ sensors. Furthermore, interferences and physical coupling between sensors are reduced compared to nested arrays [7]. The enhanced degree of freedom is based on coprime DFTFBs, which divide the spectrum equally into $M N$ bands by statistically averaging the outputs of two uniform linear arrays.

However, systematic design procedures for coprime DFTFBs were not fully studied in [12]. The extended coprime arrays with various window functions were considered in [1]. This problem is in essence a filter design problem. Given the specifications of coprime DFTFBs, the impulse responses of two DFTFBs are to be evaluated.

Similar design problems can be found in filter design literature as interpolated finite impulse response filters (IFIR filters) [5], [9], [10]. IFIR filters decompose the desired filter response into a sparse coefficient filter followed by an interpolation filter. These filters are designed jointly to minimize the number of multipliers while meeting the specification [4]. By the virtue of low complexity, IFIR filters find useful applications in IFIR radar [11], active beamforming [13], adaptive filtering [2], and low power circuit design [6].

The goal of this paper is to design coprime DFTFBs so that the performance of coprime arrays is fundamentally improved. A systematic design procedure of coprime DFTFBs, inspired by the design methods for IFIR filters, is presented. Since IFIR filters are coprime DFTFBs with $N=1$, design techniques for IFIR filters can be extended to coprime DFTFBs. With

\footnotetext{
${ }^{1}$ This work was supported in parts by the ONR grant N00014-11-1-0676, and the California Institute of Technology.
}

given specifications, the Parks-McClellan filter design algorithm is applied twice to obtain coprime DFTFBs. Practical design issues, such as transition bandwidth and overlapping between filters, are parametrized. These coprime DFTFBs with better specifications faciliate a variety of applications in array processing.

This paper is organized as follows. Coprime DFTFBs will be reviewed briefly in Section II. The design parameters are defined in Section III, where an additional parameter $\lambda$ controls the behavior in stopbands. In Section IV, optimization problems regarding $\lambda$ are proposed, relaxed, and solved in closed-form expressions. Frequency responses of our coprime DFTFB designs are simulated in Section V before concluding remarks in Section VI.

\section{REVIEW OF COPRIME DFT FILTER BANKS}

Assume that $M$ and $N$ are positive coprime integers. Consider two real coefficient FIR filters $G(z)$ and $H(z)$,

$$
G(z)=\sum_{n=0}^{N_{g}} g(n) z^{-n}, \quad H(z)=\sum_{n=0}^{N_{h}} h(n) z^{-n},
$$

where $N_{g}$ and $N_{h}$ are filter orders of $G(z)$ and $H(z)$, respectively. Coprime DFTFBs are a set of $M N$ filters defined as

$$
F_{\ell k}(z)=G\left(z^{M} W_{N}^{\ell}\right) H\left(z^{N} W_{M}^{k}\right),
$$

where $0 \leq \ell \leq N-1,0 \leq k \leq M-1$ and $W_{N}=e^{-j 2 \pi / N}$. In [12], $G(z)$ and $H(z)$ are ideal lowpass filters

$$
\begin{aligned}
& G\left(e^{j 2 \pi f}\right)= \begin{cases}1 & f \in\left(-\frac{1}{2 N}, \frac{1}{2 N}\right), \\
0 & \text { otherwise, }\end{cases} \\
& H\left(e^{j 2 \pi f}\right)= \begin{cases}1 & f \in\left(-\frac{1}{2 M}, \frac{1}{2 M}\right), \\
0 & \text { otherwise, }\end{cases}
\end{aligned}
$$

where $f$ denotes normalized frequencies. $G\left(e^{j 2 \pi f M}\right)$ and $H\left(e^{j 2 \pi f N}\right)$ have exactly $M$, and $N$ passbands, respectively. Only one of the passbands overlaps, implying $F_{\ell k}\left(e^{j 2 \pi f}\right)$ has exactly one passband with bandwidth $\frac{1}{M N}$. Assume that the passband is centered around $\frac{i}{M N}$, where $0 \leq i \leq M N-1$. Defining $\ell$ and $k$ to be such that

$$
\ell=i \bmod M, \quad k=i \bmod N,
$$

the coprime DFTFB $F_{\ell k}\left(e^{j 2 \pi f}\right)$ is then

$$
F_{\ell k}\left(e^{j 2 \pi f}\right)= \begin{cases}1 & f \in\left(\frac{i}{M N}-\frac{1}{2 M N}, \frac{i}{M N}+\frac{1}{2 M N}\right), \\ 0 & \text { otherwise. }\end{cases}
$$



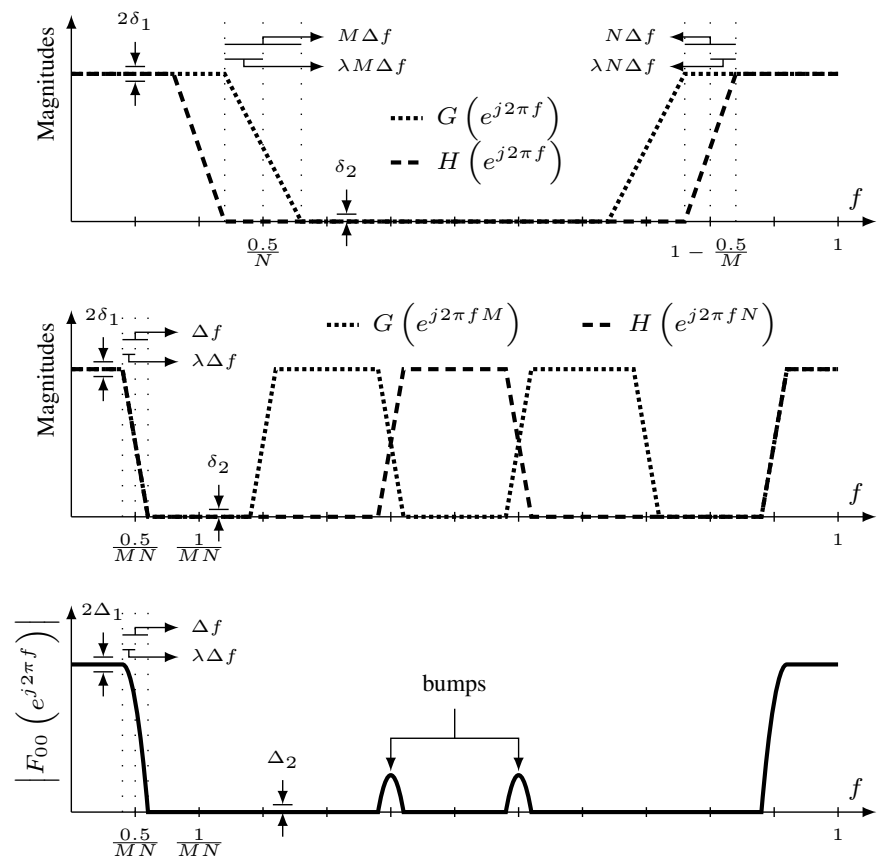

Fig. 1. An illustration of Coprime DFTFBs for $M=3$ and $N=2$. (Top) Prototype filters $G\left(e^{j 2 \pi f}\right)$ and $H\left(e^{j 2 \pi f}\right)$. (Middle) Magnitude responses of sparse coefficient filters $G\left(e^{j 2 \pi f M}\right)$ and $H\left(e^{j 2 \pi f N}\right)$. (Bottom) The first filter $\left(F_{00}\left(e^{j 2 \pi f}\right)=G\left(e^{j 2 \pi f M}\right) H\left(e^{j 2 \pi f N}\right)\right)$ in coprime DFTFBs.

The filter $F_{00}(z)$ can be regarded as a generalization of IFIR filters. Letting $N=1$ in $F_{00}(z)=G\left(z^{M}\right) H\left(z^{N}\right)$ leads to IFIR filters exactly, if we view $H(z)$ as the interpolation filter. Besides, $G(z)$ and $H(z)$, as the model filters in IFIR filters, have wider passbands compared to $F_{00}(z)$. The number of multipliers in $F_{00}(z)$ is reduced to $N_{g}+N_{h}+2$, which is similar to the low complexity of IFIR filters.

\section{DESIGN FROM SPECIFICATION}

The goal of coprime DFTFB design is to obtain $g(n)$ and $h(n)$ so that (1) is well-approximated in the sense of peak passband ripples $\Delta_{1}$ and peak stopband ripples $\Delta_{2}$. Provided with coprime integers $M, N$, filter orders $N_{g}, N_{h}$, and peak ripples $\Delta_{1}, \Delta_{2}$, we need to relate these parameters to the specifications of $G\left(e^{j 2 \pi f}\right)$ and $H\left(e^{j 2 \pi f}\right)$. Then the ParksMcClellan filter design algorithm can be directly applied to these specifications to obtain $g(n)$ and $h(n)$.

The concept of coprime DFTFB design is illustrated in Fig. 1. Two lowpass filters $G\left(e^{j 2 \pi f}\right)$ and $H\left(e^{j 2 \pi f}\right)$ are designed with transition bandwidths $M \Delta f$ and $N \Delta f$, respectively. The ideal passbands are eroded by the finite transition band with a factor $\lambda \in[0,1]$. The corresponding sparse coefficient filters $G\left(e^{j 2 \pi f M}\right)$ and $H\left(e^{j 2 \pi f N}\right)$ narrow down the transition bandwidth to $\Delta f$ but create unwanted image bands. According to [12], the undesired bands will be cancelled out in the ideal case because of coprimality of $M$ and $N$. In practice, the overlapping transition bands in $G\left(e^{j 2 \pi f M}\right)$ and $H\left(e^{j 2 \pi f N}\right)$ introduce some bumps in the stopband, as depicted in the bottom of Fig. 1. These bumps might violate stopband ripple constraints.

Next, the peak ripples for the filters $G\left(e^{j 2 \pi f}\right), H\left(e^{j 2 \pi f}\right)$, and $F_{00}\left(e^{j 2 \pi f}\right)$ are related. Without loss of generality, the peak passband ripples and the peak stopband ripples of $G\left(e^{j 2 \pi f}\right), H\left(e^{j 2 \pi f}\right)$ are set to be $\delta_{1}$ and $\delta_{2}$, as illustrated in the top plot of Fig. 1. The peak passband/stopband ripples for $F_{00}\left(e^{j 2 \pi f}\right)$ are defined as $\Delta_{1}$ and $\Delta_{2}$, respectively. Since sparse coefficient filters do not modify the ripples, $\Delta_{1}, \Delta_{2}, \delta_{1}, \delta_{2}$ are related by

$$
1-\Delta_{1}=\left(1-\delta_{1}\right)^{2}, \quad \Delta_{2}=\left(1+\delta_{1}\right) \delta_{2},
$$

where the bumps are not considered in the above formulation. Expressing $\delta_{1}$ and $\delta_{2}$ in terms of $\Delta_{1}$ and $\Delta_{2}$ yields

$$
\delta_{1}=1-\sqrt{1-\Delta_{1}}, \quad \delta_{2}=\frac{\Delta_{2}}{2-\sqrt{1-\Delta_{1}}} .
$$

If the given ripples $\Delta_{1}$ and $\Delta_{2}$ are relatively small compared to unity, the peak ripples can be approximated by $\delta_{1} \approx \Delta_{1} / 2$ and $\delta_{2} \approx \Delta_{2}$.

The transition bandwidths of $G\left(e^{j 2 \pi f}\right)$ and $H\left(e^{j 2 \pi f}\right)$ are $M \Delta f$ and $N \Delta f$, respectively. Since we know filter orders can be estimated from transition bandwidths and peak ripples by Bellanger's formula [10, pp. 57] (Kaiser's formula [10, pp. 57] will also work), $\Delta f$ can be lower-bounded by

$$
\Delta f \geq \frac{2 \log _{10}\left(\frac{1}{10 \delta_{1} \delta_{2}}\right)}{3 \min \left\{M N_{g}, N N_{h}\right\}} .
$$

Since Bellanger's formula only provides an estimate for filter orders, this lower bound does not guarantee that the resultant $G\left(e^{j 2 \pi f}\right)$ and $H\left(e^{j 2 \pi f}\right)$ meet the specifications exactly. If our design does not meet the specifications, increasing $\Delta f$ will work.

To run McClellan filter design algorithm on $G\left(e^{j 2 \pi f}\right)$ and $H\left(e^{j 2 \pi f}\right)$, the passband/stopband edges need to be specified, which are related to the parameter $\lambda$. As depicted in Fig. 1, $\lambda$ controls the amount of overlapping transition bands between $G\left(e^{j 2 \pi f M}\right)$ and $H\left(e^{j 2 \pi f N}\right)$ as well as the level of bumps in $F_{\ell k}\left(e^{j 2 \pi f}\right)$. In the next section, we will propose an optimization problem over $\lambda$ that meets the design specifications.

\section{Parameter $\lambda$ Selection}

The parameter $\lambda$ offers tradeoffs between bandwidths of the passband and the stopband. Consider the extreme cases $\lambda=1$ and $\lambda=0$. If $\lambda=1$, the bumps of $F_{00}\left(e^{j 2 \pi f}\right)$ are less than $\delta_{2}^{2}$, which satisfies stop ripple constraints. However, the passband width is minimized over all possible $\lambda$, which rejects the components around $f=0.5 /(M N)$. When $\lambda=0$, although the passband edge is maximized to be $0.5 /(M N)$, the bumps violate the stopband ripple constraints.

Our design goal is to limit the stopband ripples of $F_{00}\left(e^{j 2 \pi f}\right)$ to be always less than $\Delta_{2}$. Among all feasible solutions, the one with maximal passband edge is desired. This 
statement poses the following optimization problem:

$$
\begin{aligned}
& \lambda_{o p t}=\min _{\lambda} \lambda \quad \text { subject to } \quad\left|F_{00}\left(e^{j 2 \pi f}\right)\right| \leq \Delta_{2}, \\
& f \in\left[\frac{0.5}{M N}+(1-\lambda) \Delta f, 1-\frac{0.5}{M N}-(1-\lambda) \Delta f\right],
\end{aligned}
$$

where $\Delta f$ is fixed.

However, there is no simple solver to (4) other than bruteforce search over all possible $\lambda$. The frequency response $F_{00}\left(e^{j 2 \pi f}\right)$ and the parameter $\lambda$ need to be solved alternatively. This procedure is not practical since a simple design problem specifies $\lambda$ before designing filters.

Instead of solving (4), the optimization problem can be relaxed to approximated frequency responses $\hat{F}_{00}\left(e^{j 2 \pi f}\right)$.

$$
\begin{aligned}
& \hat{\lambda}=\min _{\lambda} \lambda \quad \text { subject to } \quad\left|\hat{F}_{00}\left(e^{j 2 \pi f}\right)\right| \leq \Delta_{2}, \\
& f \in\left[\frac{0.5}{M N}+(1-\lambda) \Delta f, 1-\frac{0.5}{M N}-(1-\lambda) \Delta f\right],
\end{aligned}
$$

where $\hat{F}_{00}\left(e^{j 2 \pi f}\right)$ might not be realizable in the FIR setting but can be written as some simple closed-form functions. Certain choices of $\hat{F}_{00}\left(e^{j 2 \pi f}\right)$ even give closed-form expression of $\hat{\lambda}$. These $\hat{\lambda}$ are simple to evaluate and close to $\lambda_{\text {opt }}$.

A fundamental question that might be raised here is the conditions on the approximated frequency responses. The only assumption in the paper is that the transition bands of $G\left(e^{f 2 \pi f}\right)$ and $H\left(e^{j 2 \pi f}\right)$ are monotonically increasing or decreasing. This is usually a valid assumption for the ParksMcClellan filter design algorithm.

In the following discussion, linear functions and $Q$ functions approximating the transition bands and the minimizer to (5), $\hat{\lambda}$, are derived.

\section{A. Linear functions}

Fig. 1 illustrates a simplified model of the transition band of $G\left(e^{j 2 \pi f M}\right)$ and $H\left(e^{j 2 \pi f N}\right)$ for $M=3$ and $N=2$. Within the transition bands, which is around $f=5 /(2 M N)$ in Fig. 1, the line segments joining the passband edges and the stopband edges are

$$
\begin{aligned}
& \hat{G}_{l i}(f)=\delta_{2}-\left(1-\delta_{1}-\delta_{2}\right) \frac{f-\frac{2.5}{M N}-(1-\lambda) \Delta f}{\Delta f}, \\
& \hat{H}_{l i}(f)=\delta_{2}+\left(1-\delta_{1}-\delta_{2}\right) \frac{f-\frac{2.5}{M N}+(1-\lambda) \Delta f}{\Delta f},
\end{aligned}
$$

where $f \in \mathcal{S}=[2.5 /(M N)-\tilde{\lambda} \Delta f, 2.5 /(M N)+\tilde{\lambda} \Delta f]$, $\tilde{\lambda}=\min (\lambda, 1-\lambda) . \hat{G}_{l i}(f)$ and $\hat{H}_{l i}(f)$ denote the linear approximation of $\left|G\left(e^{j 2 \pi f M}\right)\right|$ and $\left|H\left(e^{j 2 \pi f N}\right)\right|$, respectively. Coprime DFTFBs over $\mathcal{S}$ are estimated as

$$
\begin{aligned}
\hat{F}_{l i}(f) & =\hat{G}_{l i}(f) \hat{H}_{l i}(f) \\
& \leq \frac{1}{4}\left(\hat{G}_{l i}(f)+\hat{H}_{l i}(f)\right)^{2} \\
& =\left(\delta_{2}+\left(1-\delta_{1}-\delta_{2}\right)(1-\lambda)\right)^{2},
\end{aligned}
$$

where the inequality is a direct result of AM-GM inequality since $\hat{G}_{l i}(f)$ and $\hat{H}_{l i}(f)$ are positive over $\mathcal{S}$. Setting the upper bound in (6) less than or equal to the specification $\Delta_{2}$ gives

$$
\lambda \geq \hat{\lambda}_{l i} \triangleq \frac{1-\delta_{1}-\sqrt{\Delta_{2}}}{1-\delta_{1}-\delta_{2}} .
$$

(7) indicates that, instead of setting $\lambda=1$, we can choose $\hat{\lambda}_{l i}$ to meet the stopband ripple constraints. Note that $\hat{\lambda}_{l i}$ is less than 1 for small $\delta_{1}$ and $\delta_{2}$. As a numerical example, the specifications $M=8, N=5, N_{g}=100, N_{h}=160$, $\Delta_{1}=0.01, \Delta_{2}=0.001$ give us $\delta_{1} \approx 0.003, \delta_{2} \approx 0.001$ and $\hat{\lambda}_{l i} \approx 0.969<1$.

\section{B. Q functions}

Transition bands in actual design are more complicated than linear functions. Empirically, the linear functions are usually upper bounds of the actual transition band at most frequencies in our design examples, implying $\hat{\lambda}_{l i}$ is still far from the optimizer $\lambda_{o p t}$ in (4). We need a closed-form function which 1) has the transition behavior in a particular region, 2) approximates transition bands better, and 3) makes it easy to compute the maximal value in closed-form expressions.

$Q$ functions meet these properties. Assume that the transition bands are approximated by

$$
\hat{G}_{Q}(f)=Q\left(a_{1} f+b_{1}\right), \quad \hat{H}_{Q}(f)=Q\left(a_{2} f+b_{2}\right),
$$

where $f \in \mathcal{S}$ and the parameters $a_{1}, b_{1}, a_{2}$, and $b_{2}$ can be solved from the passband and stopband edges. Substituting the passband edge and the stopband edge, as illustrated in Fig. 1, into $\hat{G}_{Q}(f)$ gives

$$
\begin{gathered}
a_{1}\left(\frac{2.5}{M N}-\lambda \Delta f\right)+b_{1}=Q^{-1}\left(1-\delta_{1}\right) \triangleq Q_{1}, \\
a_{1}\left(\frac{2.5}{M N}+(1-\lambda) \Delta f\right)+b_{1}=Q^{-1}\left(\delta_{2}\right) \triangleq Q_{2} .
\end{gathered}
$$

Solving these equations and repeating for $\hat{H}_{Q}(f)$, we obtain

$$
\begin{aligned}
& a_{1}=\frac{Q_{2}-Q_{1}}{\Delta f}, \quad b_{1}=\frac{2.5\left(Q_{1}-Q_{2}\right)}{M N \Delta f}+(1-\lambda) Q_{1}+\lambda Q_{2}, \\
& a_{2}=\frac{Q_{1}-Q_{2}}{\Delta f}, \quad b_{2}=\frac{2.5\left(Q_{2}-Q_{1}\right)}{M N \Delta f}+(1-\lambda) Q_{1}+\lambda Q_{2} .
\end{aligned}
$$

Then an estimate for $\left|F_{00}\left(e^{j 2 \pi f}\right)\right|$ becomes $\hat{F}_{Q}(f)=$ $\hat{G}_{Q}(f) \hat{H}_{Q}(f)$ which is a product of two $Q$ functions. According to an upper bound of $Q$ functions [8], putting $Q(x) \leq \frac{1}{2} e^{-\frac{1}{2} x^{2}}$ for $x \geq 0$ into $\hat{F}_{Q}(f)$ yields

$$
\begin{aligned}
\hat{F}_{Q}(f) & \leq \frac{1}{4} e^{-\frac{1}{2}\left(a_{1} f+b_{1}\right)^{2}} e^{-\frac{1}{2}\left(a_{2} f+b_{2}\right)^{2}} \\
& =\frac{1}{4} e^{-\frac{1}{2}\left(a_{1}^{2}+a_{2}^{2}\right)\left(f+\frac{a_{1} b_{1}+a_{2} b_{2}}{a_{1}^{2}+a_{2}^{2}}\right)^{2}} e^{-\frac{1}{2} \frac{\left(a_{1} b_{2}-a_{2} b_{1}\right)^{2}}{a_{1}^{2}+a_{2}^{2}}} \\
& \leq \frac{1}{4} e^{-\frac{1}{2} \frac{\left(a_{1} b_{2}-a_{2} b_{1}\right)^{2}}{a_{1}^{2}+a_{2}^{2}}}=\frac{1}{4} e^{-\left((1-\lambda) Q_{1}+\lambda Q_{2}\right)^{2}}
\end{aligned}
$$

where the second inequality comes from the upper bound of Gaussian functions. Setting (9) less than or equal to $\Delta_{2}$ and solving for $\lambda$, we obtain

$$
\left((1-\lambda) Q_{1}+\lambda Q_{2}\right)^{2} \geq-\ln \left(4 \Delta_{2}\right)
$$




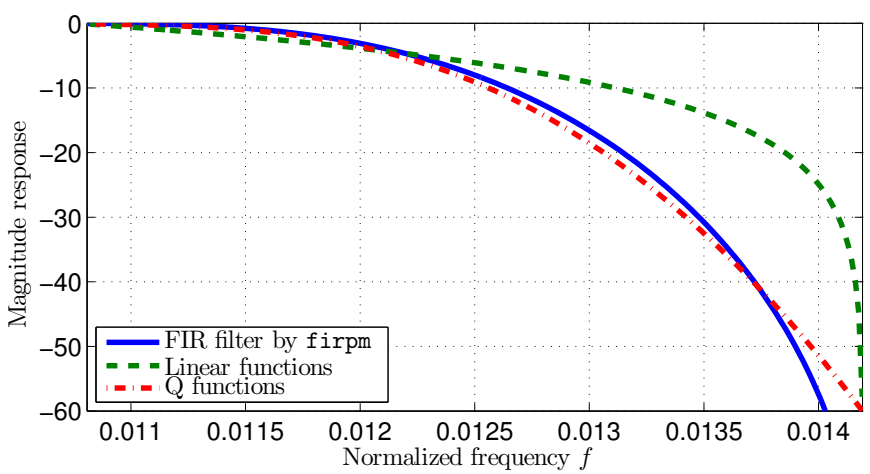

Fig. 2. A comparison among different approximations of the transition band. The FIR filter is designed by the MATLAB function $\mathrm{f}$ irpm with specification $f_{p}=0.0108, f_{s}=0.0142, \delta_{1}=0.01$, and $\delta_{2}=0.001$. The filter order is 160 .

Rearranging the inequality yields

$$
\lambda \geq \hat{\lambda}_{Q} \triangleq \frac{Q_{1}-\sqrt{-\ln \left(4 \Delta_{2}\right)}}{Q_{1}-Q_{2}},
$$

where $Q_{1} \triangleq Q^{-1}\left(1-\delta_{1}\right), Q_{2} \triangleq Q^{-1}\left(\delta_{2}\right)$, and $Q^{-1}(\cdot)$ represent inverse $Q$ functions. For the same numerical example in Sec. IV-A, we have $\hat{\lambda}_{Q}=0.86926$, which is smaller than $\hat{\lambda}_{l i}$. As a comparison, brute-force search on (4) gives $\lambda_{\text {opt }}=0.8477$.

\section{Are these approximations valid?}

The estimates for $\lambda$ are based on how well the functions approximate the actual transition band. If approximations are upper bounds of the actual transition bands, the resultant $\lambda$ are in the feasible set of (4). Conversely, lower bounds of the transition bands might lead to smaller $\lambda$ than $\lambda_{\text {opt }}$.

Fig. 2 illustrates why $\hat{\lambda}_{Q}$ is closer to $\lambda_{\text {opt }}$ than $\hat{\lambda}_{l i}$. A practical filter design with filter order 160 , passband edge $f_{p}=0.0108$, stopband edge $f_{s}=0.0142$, passband ripples $\delta_{1}=0.01$, and stopband ripples $\delta_{2}=0.001$ is simulated along with different transition band approximations. This example is actually $H\left(e^{j 2 \pi f N}\right)$ in the design example of Section V.

Linear functions offer loose upper bounds of the transition band for frequencies around $f_{s}$ according to Fig. 2. This phenomenon also confirms that $\hat{\lambda}_{l i}$ is much greater than $\lambda_{\text {opt }} . Q$ functions, however, provide better approximations of the transition band than linear functions. Hence, the relaxed optimization problem, (5) works better under the $Q$ function assumption.

\section{A Design Example}

A design example is given with $M=8, N=5, N_{g}=$ $100, N_{h}=160, \Delta_{1}=0.01$, and $\Delta_{2}=0.001$, respectively. Following the design procedure in Table $\mathrm{I}$, we obtain $\Delta f=$ 0.0036 . The frequency responses of $F_{00}\left(e^{j 2 \pi f}\right)$ are shown in Fig. 3 with different $\lambda$. This simulation shows that larger $\lambda$ eliminates bumps in the stopband but reduces the passband width at the same time. $\lambda_{\text {opt }}$ maximizes the passband width while the stopband ripple constraints are met. The proposed
TABLE I

COPRIME DFTFB DESIGN

\begin{tabular}{l}
\hline Inputs: $\left(M, N, N_{g}, N_{h}, \Delta_{1}, \Delta_{2}\right)$ \\
\hline Initialize: \\
$\delta_{1}=1-\sqrt{1-\Delta_{1}}$, \\
$\delta_{2}=\Delta_{2} /\left(2-\sqrt{1-\Delta_{1}}\right)$, \\
$\Delta f \geq 2 \log _{1} 0\left(\frac{1}{10 \delta_{1} \delta_{2}}\right) /\left(2 \min \left\{M N_{g}, N N_{h}\right\}\right)$, \\
$\lambda=\hat{\lambda}_{l i}=\left(1-\delta_{1}-\sqrt{\Delta_{2}}\right) /\left(1-\delta_{1}-\delta_{2}\right)$ \\
or $\hat{\lambda}_{Q}=\left(Q_{1}-\sqrt{-\ln \left(4 \Delta_{2}\right)}\right) /\left(Q_{1}-Q_{2}\right)$, \\
\hline Increase $\lambda$ until stopband ripples for $F_{00}\left(e^{j 2 \pi f}\right)$ are satisfied. \\
Increase $\Delta f$ until ripples for $G\left(e^{j 2 \pi f}\right)$ and $H\left(e^{j 2 \pi f}\right)$ are met. \\
Design lowpass filters $g(n)$ and $h(n)$ with specifications \\
$\quad\left(\delta_{1}, \delta_{2}, 0.5 / N-\lambda M \Delta f, 0.5 / N+(1-\lambda) M \Delta f\right)$ for $g(n)$, \\
$\quad\left(\delta_{1}, \delta_{2}, 0.5 / M-\lambda N \Delta f, 0.5 / M+(1-\lambda) N \Delta f\right)$ for $h(n)$. \\
\hline Output: $(g(n), h(n))$ \\
\hline
\end{tabular}
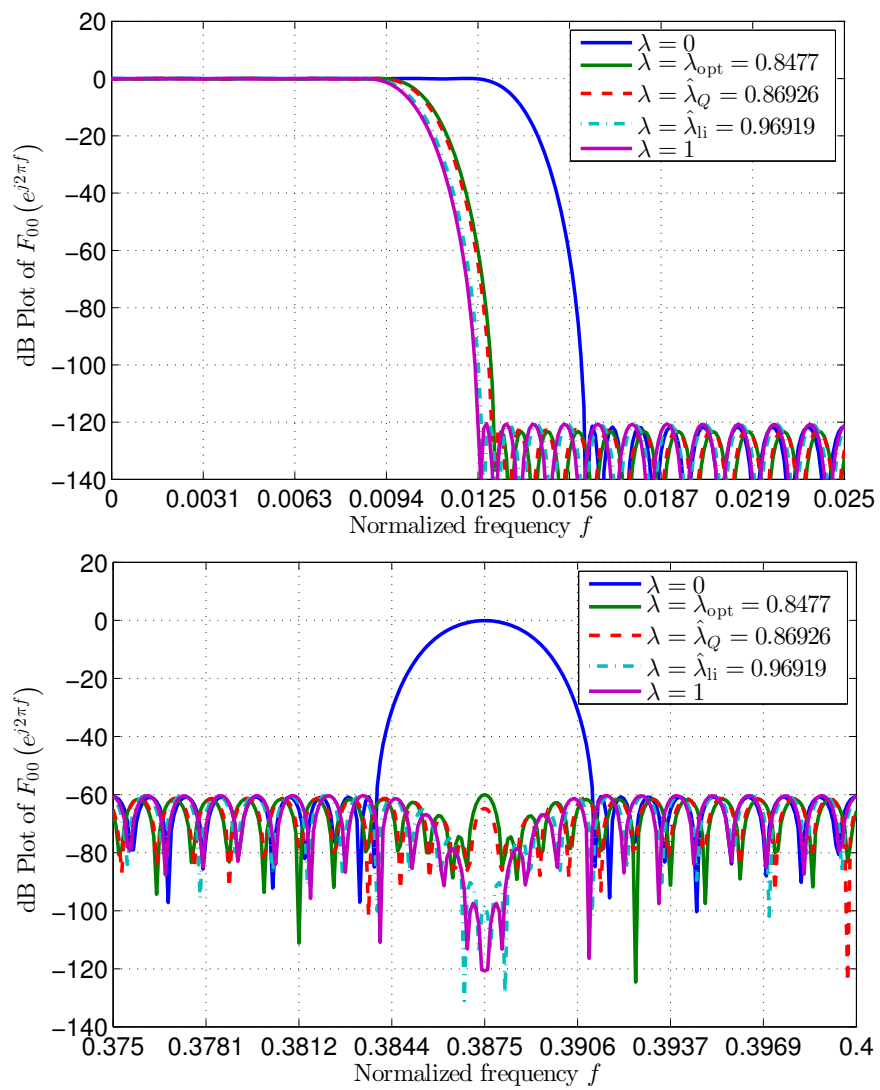

Fig. 3. A coprime DFTFB design example with $M=8, N=5, N_{g}=$ $100, N_{h}=120, \Delta_{1}=0.01$, and $\Delta_{2}=0.001 . \mathrm{dB}$ plots of $F_{00}\left(e^{j 2 \pi f}\right)$ for various $\lambda$ showing the passband behavior (top) and part of the stopband (bottom). 

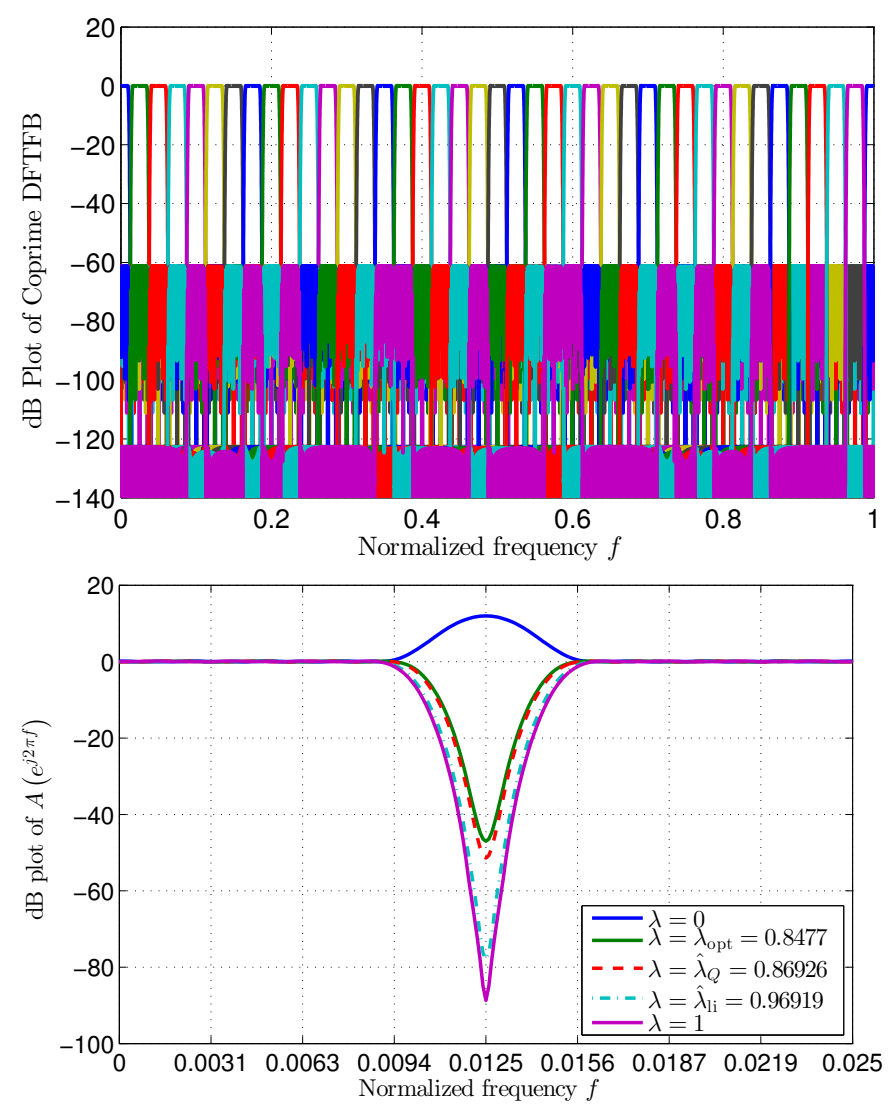

Fig. 4. Complete $M N$ bands of coprime DFTFB with the same specification as Fig. 3 and $\lambda=0.86926$. Top: The overall filter bank response. Bottom: overall amplitude response for various $\lambda$.

estimator using $Q$ functions, $\hat{\lambda}_{Q}$, yields a reasonable coprime DFTFB design closer to the optimum one, compared to $\hat{\lambda}_{l i}$ or $\lambda=1$.

So far, only the performance of single filter in the coprime DFTFB was discussed. To evaluate the overall spectrum coverage, the overall amplitude response $A\left(e^{j 2 \pi f}\right)$ is defined as

$$
A\left(e^{j 2 \pi f}\right)=\sum_{\ell=0}^{N-1} \sum_{k=0}^{M-1}\left|F_{\ell k}\left(e^{j 2 \pi f}\right)\right|,
$$

which follows the concept of amplitude distortion in quadrature mirror filter (QMF) bank design [10, Ch. 5] . In the ideal case, $A\left(e^{j 2 \pi f}\right)$ is unity almost everywhere. The error between $A\left(e^{j 2 \pi f}\right)$ and 1 is an indicator of how much the spectrum is covered by coprime DFTFBs.

On the top plot of Fig. 4, frequency responses of $M N=40$ filters cover the spectrum with 40 distinct passbands. On the bottom plot, $A\left(e^{j 2 \pi f}\right)$ is shown only around the passband. For different $\lambda, A\left(e^{j 2 \pi f}\right)$ exhibit "dips" or "bumps" around $f=0.0125$, even in the optimal solution to (4). Dips in $A\left(e^{j 2 \pi f}\right)$ imply that signals around this frequency are suppressed compared to other frequencies. If the resultant coprime DFTFBs are used in direction-of-arrival (DOA) estimation or spectrum sensing, sources at these particular DOAs/frequencies might be rejected down to the noise floor.
To resolve this problem, smaller $\lambda$ than $\lambda_{\text {opt }}$ could eliminate dips but, as a tradoff, bumps in $F_{00}\left(e^{j 2 \pi f}\right)$ will violate the stopband ripple constraints.

\section{CONCLUding REMARKS}

In this paper, we related coprime DFTFBs in array processing to the IFIR filter design. Given two coprime integers $M$, $N$, filter orders, and peak ripples, we proposed practical design steps for coprime DFTFBs. An additional parameter $\lambda$ was introduced to formulate the effect of bumps in the stopband. Closed-form expressions of $\lambda$ with different approximation models were also derived. It was verified through simulation that $\hat{\lambda}$ is close to the optimal solution $\lambda_{\text {opt }}$ to (4).

In the future, our coprime DFTFB design procedure can be applied to a variety of applications such as directionalor-arrival estimation [12], beamforming [13], and spectrum sensing in cognitive radios [3] with $O(M+N)$ sensors and $O(M N)$ degrees of freedom. For instance, in cognitive radios, spectra need to be sensed with few samples, with fast algorithms, and with high accuracy. Coprime DFTFBs resolve $O(M N)$ bands using only $O(M+N)$ samples. If designed properly, our systematic design procedure might improve the detection accuracy in cognitive radios.

\section{REFERENCES}

[1] K. Adhikari, J. R. Buck, and K. E. Wage, "Extending coprime sensor arrays to achieve the peak side lobe height of a full uniform linear array," EURASIP Journal on Advances in Signal Processing, vol. 2014, no. 1, 2014.

[2] R. C. de Lamare and R. Sampaio-Neto, "Adaptive reduced-rank MMSE filtering with interpolated FIR filters and adaptive interpolators," IEEE Signal Process. Lett., vol. 12, no. 3, pp. 177-180, March 2005.

[3] B. Farhang-Boroujeny, "Filter bank spectrum sensing for cognitive radios," IEEE Trans. Signal Process., vol. 56, no. 5, pp. 1801-1811, May 2008.

[4] A. Mehrnia and A. N. J. Willson, "On optimal IFIR filter design," in Proc. IEEE Int. Symp. Circuits and Syst. (ISCAS), vol. 3, May 2004, pp. III-133-6 Vol.3.

[5] Y. Neuvo, C.-Y. Dong, and S. K. Mitra, "Interpolated finite impulse response filters," IEEE Trans. Acoust., Speech, Signal Process., vol. 32, no. 3, pp. 563-570, Jun 1984.

[6] L. S. Nielsen and J. Sparsø, "Designing asynchronous circuits for low power: an IFIR filter bank for a digital hearing aid," Proc. IEEE, vol. 87, no. 2, pp. 268-281, Feb 1999.

[7] P. Pal and P. P. Vaidyanathan, "Nested arrays: A novel approach to array processing with enhanced degrees of freedom," IEEE Trans. Signal Process., vol. 58, no. 8, pp. 4167-4181, Aug 2010.

[8] J. G. Proakis and M. Salehi, Digital Communications, 5th ed. McGrawHill, 2008.

[9] T. Saramäki, Y. Neuvo, and S. K. Mitra, "Design of computationally efficient interpolated FIR filters," IEEE Trans. Circuits Syst., vol. 35, no. 1, pp. 70-88, Jan 1988.

[10] P. P. Vaidyanathan, Multirate Systems And Filter Banks. Pearson Prentice Hall, 1993.

[11] P. P. Vaidyanathan and P. Pal, "MIMO radar, SIMO radar, and IFIR radar: a comparison," in Proc. IEEE Asil. Conf. on Sig., Sys., and Comp., Nov 2009, pp. 160-167.

[12] P. P. Vaidyanathan and P. Pal, "Sparse sensing with co-prime samplers and arrays," IEEE Trans. Signal Process., vol. 59, no. 2, pp. 573-586, Feb 2011.

[13] P. P. Vaidyanathan and C.-C. Weng, "Active beamforming with interpolated FIR filtering," in Proc. IEEE Int. Symp. Circuits and Syst. (ISCAS), May/Jun. 2010, pp. 173-176. 